\title{
NEW INSTRUMENT FOR REMOVING METALLIC, NON-MAGNETIC INTRA-OCULAR FOREIGN BODIES*
}

BY

\author{
KAZIMIERZ GERKOWICZ \\ Ophthalmological Clinic, Medical Academy, Lublin, Poland
}

THE removal of intra-ocular foreign bodies, especially those of a non-magnetic character, is one of the major problems of ophthalmological surgery. Several excellent methods of localization have been developed and a number of useful instruments devised, but the removal of such bodies still presents great difficulty and is often a matter of chance. We have developed a device comprising a special contact forceps with a signal unit, which gives a warning when the forceps grasps the foreign body.

The forceps (Fig. 1) is of the anatomical type. On the inner side of the arms are insulating plates which prevent the two ends being brought closer together than $0.1 \mathrm{~mm}$. Thus, even when closed, the forceps constitutes a gap in an electric circuit, unless a metallic body comes between the tips. The arms are connected by wires to a signal unit, which produces an optical or acoustic signal when the circuit is closed by a foreign body.

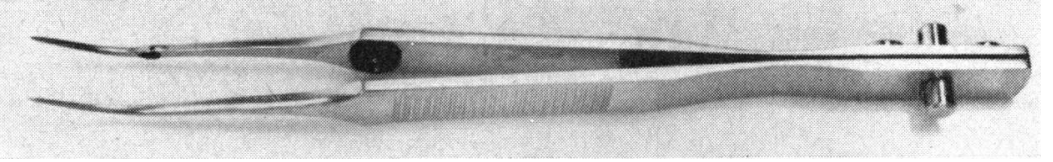

Fig. 1.-Contact forceps, actual size.

A dry-cell battery of $4.5 \mathrm{~V}$ is used as the source of a direct current feeding the forceps circuit and the signalling device, which consists of an amplifier and an incandescent lamp or a buzzer.

Experiments carried out on rabbit eyes showed that potentials above $1 \mathrm{~V}$ may produce undesirable electrolytic effects in the vitreous. For this reason the circuit potential was reduced to a few tenths of a volt, which may easily be done by means of a potentiometric voltage divider. The range of possible changes in voltage should not exceed the safety limits.

This low potential being inadequate to produce a warning signal, an amplifier is added to the signalling device. We used a two-step transistor amplifier, which comes into action a few milli-seconds after the forceps circuit has been closed.

The signal unit comprises a polarized relay (used for telegraphy, made by RFT, Germany, type 0373-001-51218, working current 5 to $10 \mathrm{~mA}$ ) and two transistors, OC71 and OC72, with an incandescent lamp, 2.5-3.5 V. A detailed circuit diagram is shown in Fig. 2 (opposite).

The unit is housed in a plastic box (Fig. 3, opposite), which also contains a measuring instrument showing the full voltage of the battery and the voltage on the potentiometer, which measures the potential present at the tips of the forceps inside the eye.

The resistors marked $\mathbf{R}_{\mathbf{1}}$ and $\mathbf{R}_{\mathbf{2}}$ in the circuit diagram should be chosen in correlation with the measuring instrument, so that potentials can be measured within ranges up to $1 \mathrm{~V}$ and up to $5 \mathrm{~V}$.

The forceps and the signal (incandescent lamp or buzzer) are connected to the amplifier by means of radio sockets and coil cords (Fig. 4, opposite).

* Received for publication July $15,1965$.

$\dagger$ Address for reprints: as above. 
FIG. 2.-Circuit diagram of two-step amplifier.

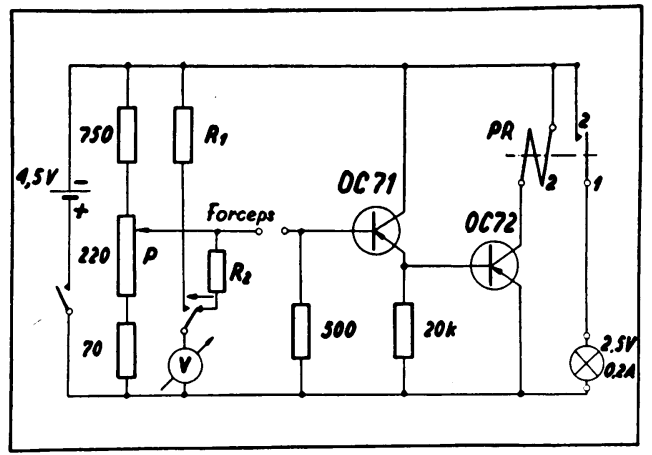

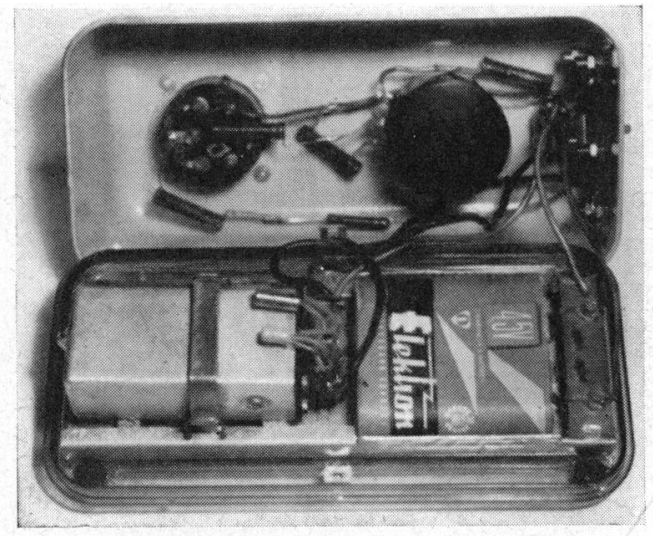

FIG. 3.-Amplifier in plastic box.

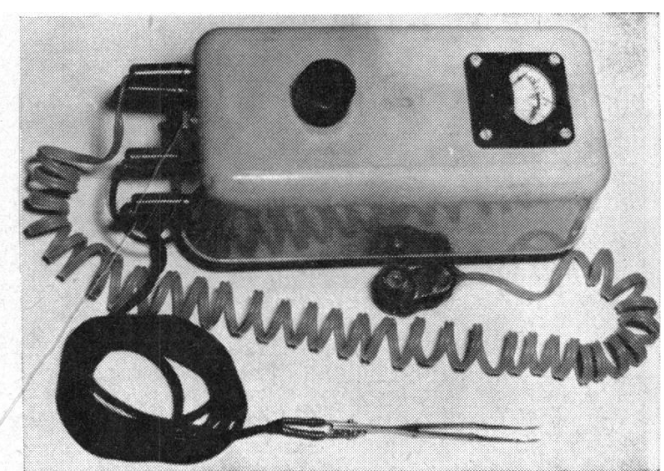

FIG. 4.-Contact forceps, amplifier, and signalling device assembled.

This signal system permits the search for an intra-ocular splinter to be based on a more objective index than the operator's sense of touch. The appearance of the signal at the moment of grasping the splinter ensures that the foreign body has been found and shows that it remains in the grip of the instrument during its withdrawal from the eyeball.

Speed in finding a foreign body depends mainly on accurate localization, but it is equally important to know exactly when contact is made. In this way repeated trauma to the eye may be avoided, and conditions for post-operative healing are much improved.

\section{Summary}

A special electrical contact forceps is described, coupled with a signalling device which marks the moment when an intra-ocular non-magnetic metallic foreign body is grasped by the forceps. Repeated introduction of the forceps into the eyeball can be thus avoided, reducing operative trauma and improving prognosis. 